\title{
7 A DEPRESSION ANXIETY STRESS SCALE EM PESSOAS COM DOENÇA RENAL CRÓNICA
}

| Luís de Sousa'; Cristina Marques-Vieira²; Sandy Severino³; Juan Correo ${ }^{4}$; José Gomes ${ }^{5}$; Helena José ${ }^{6}$ |

\section{RESUMO}

CONTEXTO: A pessoa com doença crónica está sujeita a estresse, ansiedade e depressão. A Depression Anxiety Stress Scale-21 está validada em Portugal, mas não em pessoas com doença renal crónica.

OBJETIVO: Verificar as propriedades psicométricas da versão portuguesa Depression Anxiety Stress Scale-21, em pessoas com doença renal crónica submetidas a programa de hemodiálise.

MÉTODO: Estudo metodológico. Foi obtida uma amostra representativa de 171 pessoas com doença renal cónica submetidas a hemodiálise em duas clinicas na região de Lisboa, Portugal. Foi realizada colheita de dados de maio a junho de 2015. Foram avaliadas as propriedades psicométricas: validade (constructo, convergente e discriminativa), confiabilidade ( $a$ de Cronbach) e estabilidade (teste-reteste).

RESULTADOS: O modelo dos três fatores da Depression Anxiety Stress Scale-21 não está completamente ajustado às pessoas com doença renal crónica, no entanto o modelo bidimensional "Depressão" e "Estresse/Ansiedade" ajusta-se, o que permite obter medidas confiáveis e estáveis.

CONCLUSÕES: As medidas de "Depressão" e "Estresse/Ansiedade" obtidas a partir da Depression Anxiety Stress Scale-21 são válidas e reprodutíveis em pessoas com doença renal crónical, quando se utiliza questionário e entrevista.

PALAVRAS-CHAVE: Insuficiência Renal Crónica. Depressão. Ansiedade. Estudos de validação.

\section{RESUMEN}

\section{"Depression Anxiety Stress Scale en personas con enfermedad} renal crónica”

CONTEXTO: Una persona con Enfermedad Renal Crónica está sujeta a estrés, ansiedad y depresión. La escala Depression Anxiety Stress Scale está validada en Portugal, pero no para personas con enfermedad renal crónica.

OBJETIVO: Verificar las propiedades psicométricas de la Depression Anxiety Stress Scale versión portuguesa, en personas con Enfermedad Renal Crónica que realizan hemodiálisis.

MÉTODO: Estudio metodológico. Muestra representativa con 171 personas con ERC que realizan hemodiálisis en dos clínicas de la región de Lisboa, Portugal. Recogida de datos realizada entre mayo y junio de 2015. Se evaluaron las propiedades psicométricas: validez (constructo, convergente y discriminante), confiabilidad ( $\alpha$ de Cronbach) y estabilidad (test retest).

RESULTADOS: El modelo de los tres factores de la Depression Anxiety Stress Scale no se adapta completamente a las personas con Enfermedad Renal Crónica. El modelo bidimensional "Depresión" y "Estrés/Ansiedad" se adapta a las personas con Enfermedad Renal Crónica que realizan hemodiálisis, permitiendo mediciones confiables y estables.

CONCLUSIONES: Las medidas de "Depresión" y "Estrés/Ansiedad" obtenidas mediante la DASS 21 son válidas y reproducibles en personas con Enfermedad Renal Crónica, utilizando cuestionario y entrevista.

DESCRIPTORES: Insuficiencia Renal Crónica. Depresión. Ansiedad. Estudios de validación.

\section{ABSTRACT \\ "Depression Anxiety Stress Scale in people with Chronic Kidney Disease"}

CONTEXT: The person with Chronic Kidney Disease is subjected to stress, anxiety and depression. The Depression Anxiety Stress Scale is validated in Portugal, but not for people with Chronic Kidney Disease.

OBJECTIVE: To assess the psychometric properties of the Portuguese version Depression Anxiety Stress Scale, in people with chronic kidney disease undergoing hemodialysis.

METHOD: Methodological study. A representative sample of 171 people Chronic Kidney Disease underwent hemodialysis in two clinics in the region of Lisbon, Portugal was obtained. Data was collected from May to June 2015. The psychometric properties were evaluated: Validity (construct, convergent and discriminant), reliability ( $\alpha$ Cronbach) and stability (test-retest).

RESULTS: The model of the three factors of the Depression Anxiety Stress Scale is not completely adjusted to people with Chronic Kidney Disease. The two-dimensional model "Depression" and "Stress / Anxiety" fits the person with Chronic Kidney Disease undergoing hemodialysis, which allows for reliable and stable measures.

CONCLUSIONS: The measures of "Depression" and "Stress / Anxiety" derived from the DASS 21 are valid and reproducible in people with Chronic Kidney Disease when using questionnaires and interviews.

\section{KEYWORDS: Renal Insufficiency, Chronic. Depression. Anxi- ety. Validation studies.}

Submetido em 16-07-2016

Aceite em 30-03-2017

\footnotetext{
1 Mestre em Políticas de Desenvolvimento dos Recursos Humanos, Doutorando em Enfermagem. Especialista enfermagem de reabilitação. Enfermeiro no Hospital Curry Cabral, Centro Hospitalar Lisboa Central EPE, Lisboa, Portugal Professor na Universidade New Atlântica, Lisboa, Portugal, luismmsousa@gmail.com

2 Mestre em Comportamento Organizacional e Gestão. Doutoranda em Enfermagem, Professora na Universidade Católica Portuguesa, cristina_marques@ics.lisboa.ucp.pt 3 Mestre em Gestão da Saúde. Especialista enfermagem de reabilitação. Enfermeira Hospital Curry Cabral, Centro Hospitalar Lisboa Central EPE, sandyseverino@gmail.com 4 Especialista enfermagem de reabilitação. Enfermeiro no Hospital Fernando da Fonseca. Lisboa Portugal, juanrosado2009@hotmail.es

5 Doutor em Saúde Publica. Professor Coordenador na Escola Superior de Saúde do Instituto Politécnico de Leiria. Portugal, jcrgomes@ipleiria.pt

6 Helena Maria Guerreiro José. Doutorada em Enfermagem. Professora na Escola de Saúde Multipefil. Luanda, Angola, helena.jose@multiperfil.co.ao
}

Citação: Sousa, L.M.M., Marques-Vieira, C.M.A., Severino, S. S. P., Correo, J.L.P., Gomes, J.C.R., \& José, H.M.G. (2017). A Depression Anxiety Stress Scale em pessoas com doença renal crónica. Revista Portuguesa de Enfermagem de Saúde Mental (17), 50-57. 


\section{INTRODUÇÃO}

A Doença Renal Crónica (DRC) é caracterizada pela destruição progressiva, lenta e irreversível da função renal, que condiciona a pessoa a realizar terapias de substituição renal, especificamente, a hemodiálise, a diálise peritoneal e o transplante (Ramos, Cavalgante, Braga, Aguiar \& Cavalgante, 2015). A hemodiálise consiste na extração das substâncias nitrogenadas tóxicas do sangue e na remoção de 1 a 4 litros de fluídos durante cerca de quatro horas, três vezes por semana (Frazão, Bezerra \& Lira, 2014). A DRC provoca diversas alterações na vida da pessoa e compromete física e psicologicamente, pois tem repercussões pessoais, familiares e sociais (Ramos et al. 2015).

A condição DRC e o tratamento hemodialítico são fontes de estresse permanente, que podem levar a pessoa ao isolamento social, à perda da capacidade laboral, à dificuldade parcial de locomoção e lazer, à diminuição da atividade física, à perda de autonomia, a alterações da imagem corporal, assim como, a um sentimento ambíguo entre o medo de viver e o de morrer (Vale, Souza \& Ribeiro, 2013). As pessoas submetidas a hemodiálise têm de lidar com perdas e alterações estressantes da imagem e das funções orgânicas, e como consequência tornam-se ansiosas e deprimidas (Madeiro, Machado, Bonfim, Braqueais \& Lima, 2010).

A Depression Anxiety Stress Scale (DASS) foi desenvolvida por Lovibond e Lovibond (1995) que se propuseram abranger a totalidade dos sintomas de ansiedade e depressão numa medida válida e confiável. O estudo fatorial desta escala revelou um novo fator onde foram incluídos os itens menos discriminativos das duas dimensões, a ansiedade e depressão. Estes itens foram agrupados num fator denominado "Estresse" e referiam-se a dificuldades em relaxar, tensão nervosa, irritabilidade e agitação. A versão portuguesa da escala Depression Anxiety Stress Scale com 21 itens (DASS21) confirmou ter propriedades idênticas às da versão original, e verificou-se o modelo tripartido: depressão, ansiedade e estresse (Ribeiro, Honrado \& Leal, 2004). Em outras investigações realizadas em Portugal, um estudo $(n=101)$ não evidenciou claramente a solução de três fatores como propõem os autores da versão original (Apóstolo, Mendes \& Azevedo, 2006), contudo, numa amostra mais alargada $(\mathrm{n}=1301)$ confirmou a existência do modelo tripartido (Apóstolo, Figueiredo, Mendes \& Rodrigues, 2011).
Os estudos apesentaram evidências de que, em várias culturas, nomeadamente Reino Unido (Henry \& Crawford, 2005), Estados Unidos da América (Sinclair et al., 2012), Indonésia, Singapura, Sri Lanka e Tailândia (Oei, Sawang, Goh \& Mukhtar, 2013). e Vietname (Tran, Tran \& Fisher, 2013), a DASS apresenta a estrutura tripartida e propriedades psicométricas adequadas, revelando-a como válida e fiável. Esperava-se que o modelo de três fatores se ajustasse melhor aos dados e que fornecesse evidência de validade de constructo para o modelo original da DASS (Lovibond \& Lovibond, 1995) em pessoas com DRC. Relativamente à validade convergente e divergente os estudos têm reportado correlações apropriadas entre as versões inglesas dos instrumentos DASS e a Positive and Negative Affect Schedule (PANAS) (Watson, Clark \& Tellegen, 1988), em algumas países como Estados Unidos da América (Gloster et al., 2008) e Reino Unido (Crawford et al., 2009). Correlações positivas tipicamente altas são observadas para a escala Negativa (validade convergente) e correlações negativas mais baixas são observadas para a escala Positiva (validade divergente) (Henry \& Crawford, 2005; Gloster et al., 2008; Apóstolo et al., 2011). Num estudo português a correlação com a DASS foi positiva e moderada com o afeto negativo (AN) da PANAS e limitada com a escala de afeto positivo (AP) (Apóstolo et al., 2011).

Num estudo realizado no Irão (Salimi \& Tayebi, 2010), numa amostra de 43 pessoas com DRC submetidas a hemodiálise, a maioria apresentava estresse, ansiedade de depressão. Face ao exposto, neste estudo pretendese verificar se a versão portuguesa DASS 21 (Ribeiro, Honrado \& Leal, 2004) mantém as propriedades psicométricas (validade e confiabilidade) em pessoas com DRC em programa de hemodiálise. O objetivo desta investigação é verificar as propriedades psicométricas da escala DASS-21 em pessoas com DRC.

\section{METODOLOGIA}

Estudo metodológico (Lima, 2011), realizado em duas unidades de diálise da Clínica Diaverum na região de Lisboa, de maio a junho de 2015. A população do estudo foi constituída por pessoas com DRC em programa de hemodiálise. Como critérios de inclusão estabeleceu-se: pessoas com DRC, sujeitas a tratamento hemodialítico há pelo menos seis meses, com idade igual ou superior a 18 anos e que consentiram, livre e esclarecidamente, participar no estudo. 
Foram excluídas as pessoas com défice cognitivo e doença psiquiátrica ativa. Nas duas clínicas de hemodiálise, 2253 pessoas com DRC cumpriram os critérios de inclusão (139 na Clínica 1 e 114 na Clínica 2). A amostra foi constituída pelas pessoas que cumpriam os critérios de elegibilidade e a sua seleção foi probabilística (aleatória sem reposição), considerando os critérios de elegibilidade. O cálculo da amostra com Intervalo de Confiança (IC) de 95\% e erro amostral de 5\% (Santos, s/d), designou uma amostra necessária de 192 pessoas (103 na Clínica 1 e 89 na Clínica 2). Foi feita de seguida a seleção aleatória sem reposição. Dos sujeitos selecionados na Clínica 1 , seis pessoas recusaram participar, duas foram internadas e duas desistiram, na Clínica 2, quatro pessoas recusaram participar, duas foram internadas e cinco desistiram. No final foram obtidos dados de 171 pessoas, 93 da Clínica 1 (89\%) e 78 (88\%) da Clínica 2. Para a colheita de dados foram utilizados os seguintes instrumentos: versão portuguesa da DASS-21 para medir o Estresse, Ansiedade e Depressão (Ribeiro, Honrado \& Leal, 2004), a versão portuguesa da PANAS para avaliar o AP e AN (Galinha \& Ribeiro, 2005), e ainda um instrumento para caracterização do perfil da amostra a nível sociodemográfico e clínico (idade, género, nacionalidade, escolaridade, atividade profissional, estado civil, tempo de diálise, presença de Hipertensão Arterial e de Diabetes).

A DASS-21 organiza-se em três subescalas, a da depressão, ansiedade e estresse, cada uma constituída por sete itens. Cada escala inclui vários conceitos, designadamente: Depressão e Disforia (dois itens); Desânimo, (dois itens); Desvalorização da vida (dois itens); $\mathrm{Au}$ todepreciação (dois itens); Falta de interesse ou de envolvimento (dois itens); Anedonia (dois itens); Inércia (dois itens); Ansiedade e Excitação do Sistema Autónomo (cinco itens); Efeitos Músculo Esqueléticos (dois itens); Ansiedade Situacional (três itens); Experiências Subjetivas de Ansiedade (quatro itens); Estresse Dificuldade em Relaxar (três itens); Excitação Nervosa (dois itens); Facilmente Agitado/Chateado (três itens); Irritável/Reação Exagerada (três itens); e Impaciências (três itens). As três escalas são compostas por sete itens cada, no total de 21 itens. Cada item consiste numa afirmação, que remete para sintomas emocionais negativos. Pede-se ao respondente que verifique a afirmação que lhe foi aplicada "na semana passada". Para cada frase existem quatro possibilidades de resposta, apresentadas numa escala tipo Likert.
Os respondentes avaliam a extensão em que experimentaram cada sintoma durante a última semana, numa escala de 4 pontos de gravidade ou frequência: "não se aplicou nada a mim" até "aplicou-se a maior parte das vezes”. Os resultados de cada subescala são determinados pela soma dos resultados dos sete itens. A escala fornece três classificações, uma por cada subescala, numa variação de zero a 21. As notas mais elevadas em cada escala correspondem a estados afetivos mais negativos. A consistência interna foi examinada com recurso ao Alfa de Cronbach e os resultados encontrados para a EADS foram respetivamente de 0,85 para a escala de depressão, de 0,74 para a de ansiedade e de 0,81 para a de estresse (Ribeiro, Honrado \& Leal, 2004). A escala PANAS (Watson, Clark \& Tellegen, 1988), que foi traduzida e adequada para a população portuguesa (Galinha \& Ribeiro, 2005). É constituída por duas subescalas: AP (afeto positivo) e AN (afeto negativo) com 10 itens cada, em que os itens são avaliados numa escala tipo Likert de 1 a 5. É pedido aos respondentes que indiquem em que medida sentiram cada uma das emoções (para cada um dos 20 itens) no presente momento. Quanto maior for a pontuação obtida em cada dimensão, mais presente está essa mesma dimensão, que varia entre um mínimo de 10 e um máximo de 50 pontos. Verificou-se a existência de 2 fatores tanto no estudo da versão portuguesa, tal como a escala original, com uma consistência interna com o valor do a de Cronbach de 0,86 (na original, $\alpha=0,88$ ) para a escala de AP e de 0,89 (na original, $\alpha=0,87$ ) para a escala de $A N$ e uma correlação entre os dois fatores de $-0,10$ (na original, $r=0,17)$ (Galinha \& Ribeiro, 2005).

As análises estatísticas foram realizadas com o Statistical Package for Social Sciences (SPSS) versão 20.0. Na avaliação das propriedades psicométricas, o estudo da fidedignidade, foi efetuado por meio do a de Cronbach e para avaliar a estabilidade utilizou-se o Coeficiente de Correlação Intraclasse (CCI) e Coeficiente de Correlação de Spearman-Brown (Sousa, Marque-Vieira, Carvalho, Veludo \& José, 2015) no teste-reteste (após 48 a 96 horas em 40 pessoas selecionadas aleatoriamente, 26 por questionário e 14 por entrevista). Foi adotado como valor mínimo 0,70 para uma consistência interna satisfatória (Sousa, Marque-Vieira, Carvalho, Veludo \& José, 2015). No estudo da validade, a análise fatorial exploratória (AFE) foi efetuada pelo método análise em componentes principais (ACP) com rotação obliqua e normalização kaiser (Ribeiro, Honrado \& Leal, 2004). 
A adequação foi avaliada pelo Kaiser-Meyer-Olkin (KMO) e teste de esfericidade de Bartlett. A validade convergente foi avaliada através da correlação de Pearson (r) entre as três dimensões da DASS-21 e as duas da PANAS (AP e AN). Para verificar a validade discriminante foi utilizado o teste $t$ student para amostras independentes, ou ANOVA para mais de duas amostras independentes. As variáveis categóricas foram expressas como percentual ou valor absoluto e as contínuas como média \pm desvio padrão ou mediana. Foi utilizado como ponto de corte a mediana na idade e no tempo de hemodiálise para criar variáveis categoriais. O nível de significância adotado foi de $\mathrm{p}<0,05$.

Foi concedida autorização pelos autores das versões portuguesas da DASS-21 (Ribeiro et al., 2004) e PANAS (Galinha \& Ribeiro, 2005). Este estudo foi aprovado pela Comissão de Ética da Diaverum (nº 1/2015). Todos os participantes assinaram o termo de consentimento livre e esclarecido após terem sido informados sobre a garantia de sigilo dos seus dados e do direito de desistência sem prejuízo para o próprio.

\section{RESULTADOS}

A amostra foi constituída por 171 pessoas que cumpriam os critérios de elegibilidade. O perfil sociodemográfico e de saúde encontra-se exposto na tabela 1 .

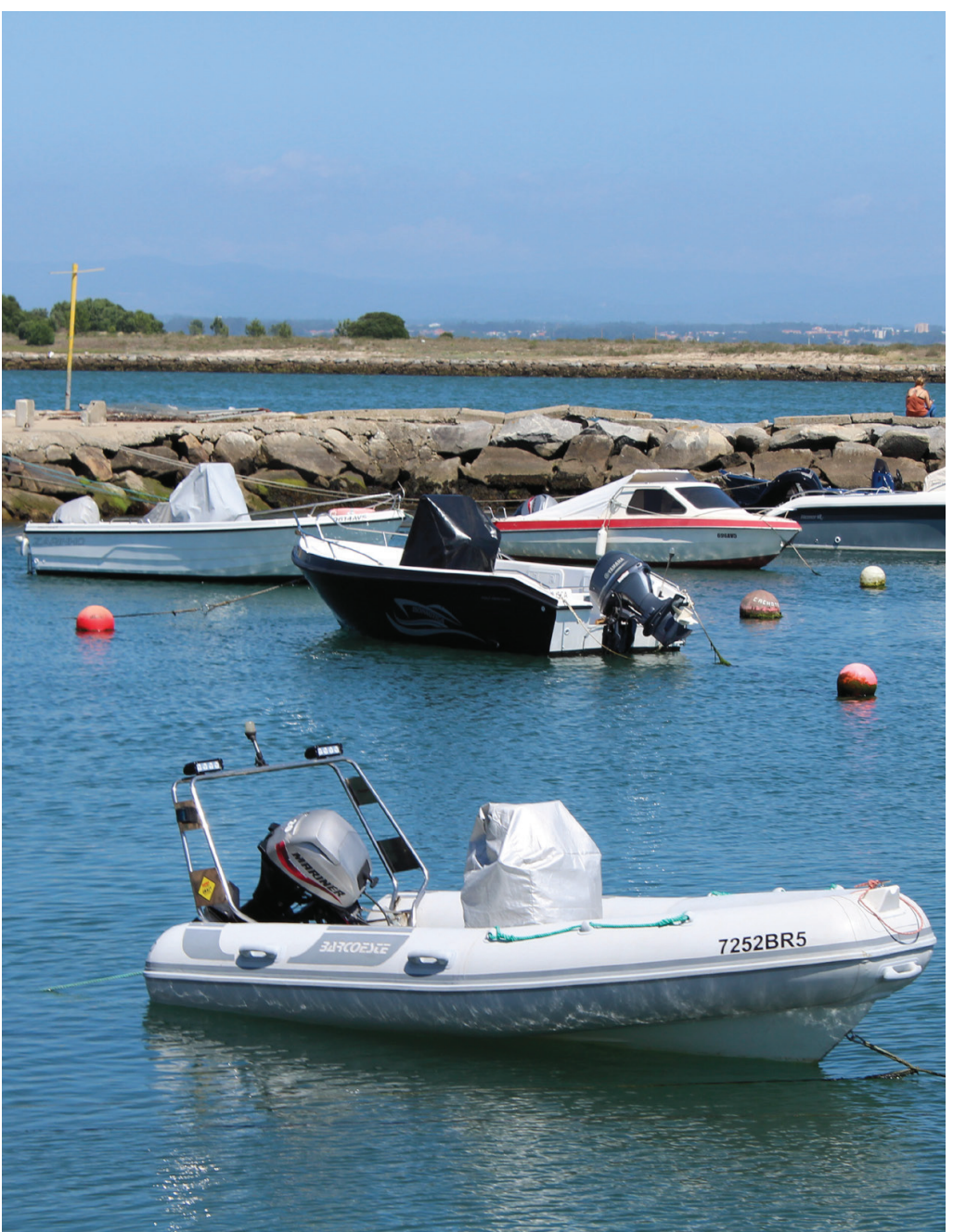

Tabela 1 - Características das pessoas com DCR avaliadas nas duas Clínicas

\begin{tabular}{|c|c|c|}
\hline Amostra & Frequência & Percentagem $\%$ \\
\hline \multicolumn{3}{|l|}{ Sexo } \\
\hline Feminino & 66 & 38,6 \\
\hline Masculino & 105 & 61,4 \\
\hline \multicolumn{3}{|l|}{ Nacionalidade } \\
\hline Portuguesa & 137 & 80,1 \\
\hline Cabo verdiana & 24 & 14,0 \\
\hline Santomense & 6 & 3,5 \\
\hline Guineense & 1 & 0,6 \\
\hline Angolana & 3 & 1,8 \\
\hline \multicolumn{3}{|l|}{ Escolaridade } \\
\hline Iliteracia & 6 & 3,6 \\
\hline 4 Ano do Ensino Básico & 72 & 42,9 \\
\hline $6^{\circ}$ Ano de Escolaridade & 31 & 18,5 \\
\hline $9^{\circ}$ Ano do Ensino Secundário & 25 & 14,9 \\
\hline $12^{\circ}$ Ano do Ensino Secundário & 19 & 11,3 \\
\hline Licenciatura & 13 & 7,7 \\
\hline Mestrado e Doutoramento & 2 & 1,2 \\
\hline \multicolumn{3}{|l|}{ Atividade profissional } \\
\hline Reformado & 125 & 76,7 \\
\hline Ativo & 38 & 23,3 \\
\hline \multicolumn{3}{|l|}{ Estado civil } \\
\hline Solteiro(a) & 44 & 25,9 \\
\hline Casado(a) & 96 & 56,5 \\
\hline Viúvo(a) & 20 & 11,8 \\
\hline Divorciado/separado & 10 & 5,9 \\
\hline \multicolumn{3}{|l|}{ Hipertensão Arterial } \\
\hline Não & 64 & 37,9 \\
\hline Sim & 105 & 62,1 \\
\hline \multicolumn{3}{|l|}{ Diabetes mellitus } \\
\hline Não & 124 & 72,9 \\
\hline Sim & 46 & 27,1 \\
\hline
\end{tabular}

A média de idade da amostra é de aproximadamente 60 anos $( \pm 14,34)$. A maioria são homens $(61 \%)$, de nacionalidade portuguesa $(80,1 \%)$, com 4 anos de escolaridade $(42,9 \%)$, estão reformados $(76,7 \%)$ e são casados $(56,5 \%)$. Relativamente aos dados de saúde, os sujeitos da amostra fazem hemodiálise há 72,17 meses $( \pm 54,23)$, $62,1 \%$ apresentam hipertensão arterial e $27,1 \%$ diabetes.

\section{Validade}

A análise fatorial exploratória (AFE) $(\mathrm{KMO}=0,91$; teste de esfericidade de Bartlett $\chi 2$ [210] 1714,785, $\mathrm{p}<0,001)$ apresentou uma solução com três fatores, que foi responsável por $53,40 \%$ da variância explicada. Todos os itens foram carregados nos fatores, com cargas fatoriais adequadas, ou seja, $>0,4$ (tabela 2 ). 
Contudo, não evidenciou claramente a solução dos três fatores. Relativamente à subescala de depressão, 7 itens apresentam a sua maior carga fatorial, entre 0,68 e 0,8 , no fator 1 . No entanto o item 5 satura no fator $3(0,73)$ e o 18 , relacionado com o estresse, saturou no fator $1(0,68)$. No "estresse" os itens $8,11,12,14$ saturam entre $-0,57$ e $-0,73$ no fator 2, mas o item 1 e 6 saturam no fator 3 (respetivamente 0,62 e 0,66). Os itens 2, 4, 7 da subescala de ansiedade saturam entre 0,53 e 0,71 no fator 3 , mas os restantes itens $(9,15,19,20)$ distribuem as respetivas cargas fatoriais pelos outros dois fatores. Dado que estes resultados põem em causa uma estrutura tridimensional, optou-se por uma solução ortogonal forçada para dois fatores, suprimindo as cargas fatoriais inferiores a 0,30 que explicam respetivamente $40,8 \%$ e $6,9 \%$, ou seja, 47,7 \% da variância total (Tabela 2).

Tabela 2 - Análise fatorial exploratória da versão portuguesa da DASS-21

\begin{tabular}{|c|c|c|c|c|c|}
\hline & \multicolumn{3}{|c|}{ Modelo 1} & \multicolumn{2}{|c|}{ Modelo 2} \\
\hline & F1 DASS-D & F2 DASS-S & F 3 DASS-A & F 1 DASSS-A & F 2 DASS-D \\
\hline $\begin{array}{l}\text { 1. Tive dificuldade em me acalmar } \\
\text { 2. Senti a minha boca seca } \\
\text { 3. Não consegui sentir nenhum sentimento positivo } \\
\text { 4. Senti dificuldade em respirar } \\
\text { 5. Tive dificuldade em tomar iniciativa para fazer as coisas } \\
\text { 6. Tive tendência a reagir em demasia em determinadas } \\
\text { situações } \\
\text { 7. Senti tremores (por ex., nas mãos) } \\
\text { 8. Senti que estava a utilizar muita energia nervosa } \\
\text { 9. Preocupei-me com situações em que podia entrar em } \\
\text { pânico e fazer figura ridícula } \\
\text { 10. Senti que não tinha nada a esperar do futuro } \\
\text { 11. Dei por mim a ficar agitado } \\
\text { 12. Senti dificuldade em relaxar } \\
\text { 13. Senti-me desanimado e melancólico } \\
\text { 14. Estive intolerante em relação a qualquer coisa que me } \\
\text { impedisse de terminar aquilo que estava a fazer } \\
\text { 15. Senti-me quase a entrar em pânico } \\
\text { 16. Não fui capaz de ter entusiasmo por nada } \\
\text { 17. Senti que não tinha muito valor como pessoa } \\
\text { 18. Senti que por vezes estava sensível } \\
\text { 19. Senti alterações no meu coração sem fazer exercício físico } \\
\text { 20. Senti-me assustado sem ter tido uma razão para isso } \\
\text { 21. Senti que a vida não tinha sentido }\end{array}$ & $\begin{array}{l}0,69 \\
0,52 \\
0,46 \\
0,74 \\
0,57 \\
\\
0,60 \\
0,73 \\
0,79 \\
0,68 \\
0,48 \\
0,61 \\
0,80\end{array}$ & $\begin{array}{l}-0,73 \\
-0,57 \\
-0,45 \\
-0,67 \\
-0,75 \\
-0,42 \\
\\
-0,56 \\
-0,71 \\
-0,40\end{array}$ & $\begin{array}{l}0,62 \\
0,71 \\
0,58 \\
0,73 \\
0,66 \\
\\
0,53 \\
0,57 \\
0,44 \\
0,55 \\
0,63 \\
0,43\end{array}$ & $\begin{array}{c}0,73 \\
0,60 \\
0,41 \\
0,6 \\
0,57 \\
0,35 \\
0,32 \\
0,44 \\
0,59\end{array}$ & $\begin{array}{l}0,62 \\
0,32 \\
0,58 \\
0,34 \\
\\
0,42 \\
0,59 \\
0,75 \\
0,50 \\
0,30 \\
0,37 \\
0,77\end{array}$ \\
\hline $\begin{array}{l}\text { Números próprios (Eigenvalue) } \\
\text { Variância explicada (\%) } \\
\text { a de Cronbach }\end{array}$ & $\begin{array}{c}8,5724 \\
0,8 \\
0,86\end{array}$ & $\begin{array}{c}1,425 \\
6,8 \\
0,86\end{array}$ & $\begin{array}{c}1,224 \\
5,8 \\
0,75\end{array}$ & $\begin{array}{c}8,5724 \\
0,8 \\
0,88\end{array}$ & $\begin{array}{c}1,425 \\
6,9 \\
0,86\end{array}$ \\
\hline
\end{tabular}

Legenda: $\mathrm{F}=$ Fator

O fator 1 corresponde ao estresse e ansiedade e o fator 2 à depressão. O item 18 do estresse saturou no fator 2 (depressão). O item 6 tem pesos fatoriais muito similares nos dois fatores. Esta última solução parece ser menos problemática. O Coeficiente $\alpha$ de Cronbach para a escala geral DASS 21 foi de 0,92, para a "Depressão" foi de 0,86, para o "Estresse/Ansiedade" foi de 0,88.

No estudo da validade convergente, efetuaram-se as correlações entre os instrumentos DASS-21 e entre a DASS Depressão, DASS Ansiedade/Estresse e PANAS (tabela 3).

Tabela 3 - Validade Concorrente e confiabilidade das medidas

\begin{tabular}{|l|c|c|c|c|c|}
\hline a de Cronbach $(\alpha)$ & DASS 21 & DASS S/A & DASS D & AP & \\
\hline DASS 21 & $(0,92)$ & & & & \\
\hline DASS S/A & $0,95^{\star *}$ & $(0,88)$ & & \\
\hline DASS D & $0,91^{\star *}$ & $0,73^{\star *}$ & $(0,86)$ & & \\
\hline AP & 0,01 & $-0,06$ & 0,05 & $(0,86)$ & \\
\hline AN & $0,50^{* *}$ & $0,47^{* *}$ & $0,44^{* *}$ & 0,09 & $(0,88)$ \\
\hline
\end{tabular}

Legenda: ${ }^{*}<0,05 ;{ }^{* *} \mathrm{p}<0,0001$; AP=Afeto Positiva; NA=Afeto Negativo; DASS-21=Pontuação global; DASS-D=Depressão; DASS-S/A=Estresse e Ansiedade. 
As escalas correlacionam entre 0,44 e 0,50 com AN (todos $\mathrm{p}<0,001$ ). As correlações com AP foram fracas e não significativas. As inter-correlações das subescalas da DASS-21 são positivas e fortes.

Observa-se nos resultados referentes à validade discriminante, com o objetivo de identificar se a DASS 21 é capaz de diferenciar a "depressão" e "estresse/ansiedade" nas variáveis género, idade, nacionalidade, atividade profissional, presença de hipertensão arterial (tabela 4), que a DASS na "estresse/ansiedade" e "depressão" consegue discriminar o género.

Tabela 4 - Validade discriminante da DASS 21

\begin{tabular}{|l|l|l|}
\hline $\begin{array}{l}\text { Características da } \\
\text { Amostra }\end{array}$ & DASS-D & DASS-S/A \\
\hline Género & $4,3( \pm 4,2)^{\star}$ & $5,5( \pm 5,6)^{\star *}$ \\
\hline Masculino & $6,1( \pm 5,4)$ & $8,6( \pm 7,1)$ \\
\hline Feminino & \multicolumn{2}{|l|}{} \\
\hline Idade & $4,4( \pm 4,7)$ & $6,5( \pm 6,2)$ \\
\hline Inferior a 63 anos & $6,9( \pm 6,7)$ \\
\hline Superior a 63 anos & $5,5( \pm 4,8)$ & $6,8( \pm 6,2)$ \\
\hline Atividade profissional & $6,2( \pm 5,9)$ \\
\hline Aposentado & $5,4( \pm 4,8)$ & \\
\hline Ativo & $3,7( \pm 4,5)$ & $5,4( \pm 5,3)^{\star}$ \\
\hline Hipertensão arterial & $7,5( \pm 6,8)$ \\
\hline Não & $4,1( \pm 4,5)$ & \\
\hline Sim & $5,5( \pm 4,8)$ & \\
\hline
\end{tabular}

Legenda: ${ }^{*}<0,05 ;{ }^{*} \mathrm{p}<0,01$; DASS-D=Depressão; DASS S/A=Estresse e Ansiedade

As mulheres apresentam níveis mais elevados de ansiedade/estresse e depressão. E por último, o "estresse/ansiedade" discriminam as pessoas com hipertensão. As pessoas com níveis de estresse e ansiedade mais elevados apresentam hipertensão arterial.

\section{Confiabilidade e estabilidade}

$\mathrm{Na}$ análise das propriedades psicométricas a reprodutibilidade da DASS 21, verificada através do coeficiente de Cronbach, na escala total, variou de 0,91 a 0,92 após a exclusão de cada um dos itens. $\mathrm{O} \alpha$ de Cronbach na segunda avaliação foi de 0,92. Na DASS “estresse e ansiedade" o a de Cronbach variou de 0,87 a 0,89 e na DASS "depressão" variou entre 0,84 a 0,87 .

$\mathrm{Na}$ avaliação da estabilidade (teste-reteste), os dados obtidos por instrumento de medida $(n=26)$, apresentaram um a de Cronbach global na primeira avaliação de 0,96 e na segunda de 0,96 . O coeficiente de correlação de Spearman-Brown que foi de 0,84 e o CCI que foi de 0,97 (IC 95\%; 0,95 a 0,98, p<0,001).
Por entrevista $(n=14)$ o a de Cronbach global na primeira avaliação foi de 0,75 e segunda avaliação foi de 0,89 . O coeficiente de correlação de Spearman-Brown foi de 0,38 e o CCI foi de 0,87 (IC95\%; 0,75 a 0,95, p<0,001), o que demonstra que houve uma boa consistência e estabilidade entre avaliações, tanto por meio de questionário autoreportado, como por entrevista.

\section{DISCUSSÃO}

Ao nível estrutural, a solução de dois fatores, revela uma melhor organização dos dados, embora um dos 14 itens das subescalas de "ansiedade/estresse" apresentem o seu peso principal fora da dimensão que agrupa os respetivos itens. É ainda problemático o item 6 que tem pesos fatoriais idênticos nos dois fatores. Os dados são diferentes dos encontrados nos estudos com versões da DASS-21 na língua inglesa (Henry \& Crawford, 2005; Sinclair et al., 2012) e em outras culturas (Oei et al., 2013; Tran et al., 2013) que apresentaram evidências de uma estrutura de três fatores. A solução de dois fatores foi relatada numa versão portuguesa desta escala (Apóstolo et al., 2006), em que um dos sete itens da subescala de "depressão" e dois dos 14 itens das subescalas de "ansiedade/estresse" apresentaram o seu peso principal fora da dimensão que agrupa os respetivos itens. Por outro lado, um dos sete itens da subescala de "depressão" e quatro de "ansiedade/estresse" saturaram em ambos os fatores. Neste estudo, a variância explicada para a solução dos dois fatores foi inferior à do estudo que apresentou a solução de dois fatores com $58,54 \%$ (Apóstolo et al., 2006). Contudo, o estudo do autor original (Lovibond \& Lovibond, 1995) revelou que os três fatores explicava $41,3 \%$ da variância, claramente inferior ao verificado neste estudo (47,7\%).

$\mathrm{Na}$ validade convergente da DASS-21 verificou-se uma correlação com a PANAS positiva e moderada na escala de AN (significativa), e limitada (não significativa) com a escala de AP. Os resultados na validade convergente são similares a um estudo português (Apóstolo et al., 2011), em que se verificou uma correlação positiva e moderada com a escala de AN, e negativa e limitada com a escala de AP. Num estudo realizado no Reino Unido verificou-se uma correlação positiva e moderada com a escala de AN, e negativa moderada com a escala de AP (Henry \& Crawford, 2005). No presente estudo obtevese apoio para a validade externa de medida, apenas com a subescala de AN. As duas dimensões da DASS-21 em pessoas com DRC conseguem discriminar pelo sexo e quando apresentam hipertensão arterial. 
Relativamente ao sexo foram relatados semelhanças num estudo português (Apóstolo et al., 2011), em que se verificou que as mulheres apresentavam pontuações mais altas que os homens em todas as subescalas.

Neste estudo os valores do a de Cronbach revelaram a confiabilidade da escala e são comparáveis aos encontrados nos restantes estudos feitos com a DASS-21 (Henry \& Crawford, 2005; Sinclair et al., 2012; Oei et al., 2013; Tran et al., 2013), em que os valores foram respetivamente: depressão $(0,82,0,91,0,86$ e 0,72$)$; ansiedade $(0,90,0,80,0,81$ e 0,77$)$ e estresse $(0,93,0,84,0,70$ e $0,70)$. Além disso, resultados dos estudos com a versão portuguesa da DASS-21 (Pais-Ribeiro et al., 2004; Apóstolo et al., 2011) apresentam valores de a de Cronbach como: depressão $(0,85$ e 0,88$)$, ansiedade $(0,74$ e 0,84$)$ e estresse $(0,81$ e 0,90$)$. Os resultados do autor original (DASS-42) (Lovibond \& Lovibond, 1995), são respetivamente para a depressão, ansiedade e estresse de 0,91, 0,81 e 0,89 . No estudo da estabilidade, com o teste-reteste, revelou ser uma medida reprodutível em ambas as modalidades de recolha de dados (questionário ou entrevista).

Estes resultados revelam diferenças na estrutura quando comparadas com a versão portuguesa (Ribeiro et al., 2004) e com a escala original (Lovibond \& Lovibond, 1995), mas assemelha-se a uma outra versão portuguesa que apresenta uma solução bifatorial (Apóstolo et al., 2006). Devido a estas discrepâncias, recomenda-se a replicação do estudo, nesta amostra, de modo a confirmar um modelo ajustado que revele a estrutura tridimensional do modelo proposto pelo autor da escala original (Lovibond \& Lovibond, 1995). Este estudo utilizou uma amostra representativa de pessoas com DRC em programa de diálise no entanto, em futuras pesquisas, seria importante realizar uma análise fatorial confirmatória nesta população específica.

\section{CONCLUSÕES}

O modelo dos três fatores da DASS-21 não está completamente ajustado às pessoas com DRC. Os resultados deste estudo não corroboram a validade da versão portuguesa da DASS-21, nesta população específica. No entanto, o modelo bidimensional "depressão" e "estresse/ ansiedade" ajusta-se à pessoa com DRC em hemodiálise, o que permite obter medidas confiáveis, reprodutíveis e válidas para “depressão" e "estresse/ansiedade”, quando utilizada sob a forma de questionário e de entrevista.

\section{REFERÊNCIAS BIBLIOGRÁFICAS}

Apóstolo, J. L. A., Figueiredo, M. H., Mendes, A. C., \& Rodrigues, M. A. (2011). Depression, anxiety and stress in primary health care users. Revista Latino-Americana de Enfermagem, 19(2), 348-353. doi: 10.1590/S010411692011000200017.

Apóstolo, J. L. A., Mendes, A. C., \& Azeredo, Z. A. (2006). Adaptation to Portuguese of the depression, anxiety and stress scales (DASS). Revista Latino-Americana de Enfermagem, 14(6), 863-871. doi: 10.1590/ S0104-11692006000600006.

Crawford, J. R., Garthwaite, P. H., Lawrie, C. J., Henry, J. D., MacDonald, M. A., Sutherland, J., \& Sinha, P. (2009). A convenient method of obtaining percentile norms and accompanying interval estimates for selfreport mood scales (DASS, DASS-21, HADS, PANAS, and sAD). British Journal of Clinical Psychology, 48(2), 163-180. doi: 10.1348/014466508X377757.

Frazão, C. M. F.Q., Bezerra, C. M. B., \& Lira, A. L. B.C. (2014). Changes in the self-concept mode of women undergoing hemodialysis: a descriptive study. Online Brazilian Journal of Nursing, 13(2), 219-26.

Galinha, I. C., \& Ribeiro, J. L. P. (2005). Contribuição para o estudo da versão portuguesa da Positive and Negative Affect Schedule (PANAS): II-Estudo psicométrico. Análise Psicológica, 23(2), 219-227.

Gloster, A. T., Rhoades, H. M., Novy, D., Klotsche, J., Senior, A., Kunik, M.,... \& Stanley, M. A. (2008). Psychometric properties of the Depression Anxiety and Stress Scale-21 in older primary care patients. Journal of Affective Disorders, 110(3), 248-259. doi: 10.1016/j. jad.2008.01.023.

Henry, J. D., \& Crawford, J.R. (2005). The short-form version of the Depression Anxiety Stress Scales (DASS-21): Construct validity and normative data in a large nonclinical sample. British Journal of Clinical Psychology, 44(2), 227-239. doi: 10.1348/014466505X29657.

Lima, D. V. M. (2011). Research design: a contribution to the author. Online Brazilian Journal of Nursing, 10(2). Disponível em: http://www.objnursing.uff.br/index.php/nursing/article/view/3648/pdf_1. 
Lovibond, P. F., \& Lovibond, S. H. (1995). The structure of negative emotional states: Comparison of the Depression Anxiety Stress Scales (DASS) with the Beck Depression and Anxiety Inventories. Behaviour Research and Therapy, 33(3), 335-343. doi:10.1016/00057967(94)00075-U.

Madeiro, A. C., Machado, P. D. L. C., Bonfim, I. M., Braqueais, A. R., \& Lima, F. E. T. (2010). Adesão de portadores de insuficiência renal crônica ao tratamento de hemodiálise. Acta Paulista de Enfermagem, 23(4), 54651. doi: 10.1590/S0103-21002010000400016.

Oei, T. P., Sawang, S., Goh, Y. W., \& Mukhtar, F. (2013). Using the depression anxiety stress scale 21 (DASS-21) across cultures. International Journal of Psychology, 48(6), 1018-1029. doi: 10.1080/00207594.2012.755535.

Ramos, I. C., Cavalcante, L.P., Braga, V. A. B., Aguiar, M. I. F., \& Cavalcante, M. B. D. P. T. (2015). Existential workshops: soft technology in nursing care for adolescents on hemodialysis. Online Brazilian Journal of Nursing, 14(1), 85-94.

Ribeiro, J. L. P., Honrado, A. A. J. D., \& Leal, I. P. (2004). Contribuição para o estudo da adaptação portuguesa das escalas de ansiedade, depressão e stress (EADS) de 21 itens de Lovibond e Lovibond. Psicologia, Saúde \& Doenças, 2, 229-239.

Santos, G.E.O. (s/d). Cálculo amostral: calculadora online. [citado 2015 Maio 1]. Disponível em: http://www. calculoamostral.vai.la.
Salimi, S. H. \& Tayebi, (2010). A survey on relationships between mental health related factors (stress, depression, anxiety) and marital satisfaction in hemodialysis patients. Nephro-Urology Monthly, 2(02), 335-344.

Sinclair, S. J., Siefert, C. J., Slavin-Mulford, J. M., Stein, M. B., Renna, M., \& Blais, M. A. (2012). Psychometric evaluation and normative data for the depression, anxiety, and stress scales-21 (DASS-21) in a nonclinical sample of US adults. Evaluation \& the Health Professions, 35(3), 259-279. doi: 10.1177/0163278711424282.

Sousa, L. M. M., Marques-Vieira, C. M. A., Carvalho, M. L., Veludo, F., \& José, H. M. G. (2015). Fidelidade e validade na construção e adequação de instrumentos de medida. Enformação, 5, 25-32. Disponível em: http:// www.acenfermeiros.pt/index.php?id1=15\&id2=9.

Tran, T. D., Tran, T., \& Fisher, J. (2013). Validation of the depression anxiety stress scales (DASS) 21 as a screening instrument for depression and anxiety in a rural community-based cohort of northern Vietnamese women. BMC Psychiatry, 13(1), 24. doi:10.1186/1471244X-13-24.

Valle, L. D. S., Souza, V. F. D., \& Ribeiro, A. M. (2013). Estresse e ansiedade em pacientes renais crônicos submetidos à hemodiálise. Estudos de Psicologia, 30(1), 131-138. doi: 10.1590/S0103-166X2013000100014.

Watson, D., Clark, L. A., \& Tellegen, A. (1988). Development and validation of brief measures of positive and negative affect: the PANAS scales. Journal of Personality and Social Psychology, 54(6), 1063-70. 